EXTENDED REPORT

\title{
The relative contributions of each subset of ocular infiltrated cells in experimental choroidal neovascularisation
}

\author{
C Tsutsumi-Miyahara, K-H Sonoda, K Egashira, M Ishibashi, H Qiao, T Oshima, T Murata, \\ M Miyazaki, I F Charo, S Hamano, T Ishibashi
}

Br J Ophthalmol 2004;88:1217-1222. doi: 10.1136/bjo.2003.036392

See end of article for authors' affiliations ....................

Correspondence to: Dr K-H Sonoda, Department of Ophthalmology, Graduate School of Medical

Sciences, Kyushu University, 3-1-1 Maidashi, Higashi-Ku, Fukuoka, Japan 8128582; sonodak@ med.kyushu-u.ac.jp

Accepted for publication 1 February 2004

\begin{abstract}
Aim: Choroidal neovascularisation (CNV) is a major cause of blindness in adults. The aim of this study was to investigate the role of infiltrating cells in the development of experimental CNV.

Methods: CNV was induced in C57BL/6 (B6) mice by laser photocoagulation (PC). After PC, the numbers of each subset of infiltrated cells were analysed by flow cytometry at multiple time points. Each subset (except for macrophages) was depleted by the specific antibodies in vivo. Thereafter, the area of CNV was compared between the control B6 mice and the specific antibody treated mice 7 days after PC. The CNV formation in neutrophil depleted CC chemokine receptor-2 (CCR2) knockout mice was also examined to minimise the effects of macrophages.

Results: In the early phase of CNV formation, a large number of neutrophils and macrophages infiltrated to the eyes. Natural killer (NK) cells and T lymphocytes were barely detected while no B lymphocytes were detected. The CNV areas did not significantly change compared between the control B6 mice and the specific antibody treated mice. However, the neutrophil depleted CCR2KO mice resulted in a reduction of CNV.

Conclusion: Although lymphocytes and NK cells had little effect on CNV formation, neutrophils partially contributed to CNV in the absence of macrophages.
\end{abstract}

\section{MATERIALS AND METHODS \\ Mice}

Female 8 week old mice were used in all experiments. C57BL/ 6 (B6) mice were purchased from Japan SLC (Shizuoka, Japan) and kept under specific pathogen free conditions at Kyushu University. CCR2 knockout (KO) mice and wild type (WT) CCR2 (+/+) mice were generated by mating homozygous mice of the same genetic background (hybrid of C57BL/6 and $129 / \mathrm{Sv}) .{ }^{16}$ All treatments of the animals conformed to the ARVO statement for the use of animals in ophthalmic and vision research.

\section{Antibodies and reagents}

The following reagents were used for the flow cytometric studies. Cy-chrome conjugated anti-mouse CD45 mAb (clone name; 30-F11), FITC conjugated anti-mouse Ly-6G (Gr-1) $\mathrm{mAb}$ (clone name; RB6-8C5), Cy-chrome conjugated antimouse TCR $\beta$ chain mAb (clone name; H57-597) and biotin conjugated anti-mouse NKl. I mAb (clone name; PK136) were purchased from BD PharMingen (San Diego, CA, USA). FITC conjugated anti-mouse CDI9 mAb (clone name; MB19-1) was purchased from eBioscience (San Diego, CA, USA). Biotin conjugated anti-F4/80 mAb (clone name; A3-1) was purchased from Caltag Lab (Burlingame, CA, USA). Streptavidin/R-PE was purchased from Molecular Probes (Oregon, USA).

The Abs used for isotype controls were as follows: Cychrome conjugated Rat IgG2b (clone name; A95-1) for antimouse CD45 mAb, FITC conjugated Rat IgG2b (clone name; A95-1), for anti-mouse Ly-6G (Gr-1) mAb, Cy-chrome conjugated Hamster IgG group2 (clone name; Ha4/8) for anti-mouse TCR $\beta$ chain mAb, biotin conjugated Mouse IgG2a (clone name;G155-178) for anti-mouse NKl. I mAb and FITC conjugated Mouse IgA (clone name; M18-254) for anti- mouse CD19 mAb were purchased from BD PharMingen (San Diego, CA, USA). Biotin conjugated rat IgG2b (clone name; R2bl5) for anti-F4/80 mAb was purchased from Caltag Lab (Burlingame, CA, USA).

The antibody preparations for in vivo were as follows: anti-mouse-granulocyte antibody, anti-Gr-l mAb is a rat

Abbreviations: $A M D$, age related macular degeneration; $C N V$, choroidal neovascularisation; NK, natural killer cells; PC, photocoagulation; RPE, retinal pigmented epithelium 
immunoglobrin G2b monoclonal antibody specific for murine neutrophilic and eosinophilic granulocytes. ${ }^{17}{ }^{18}$ Five week old female SCID mice were given intraperitoneal injections of pristane purchased from Sigma (St. Louis, MO). Ten days later, the mice received intraperitoneal inoculations of hybridoma cells (RB6-8C5) which was kindly provided by Dr F Sendo (Yamagata University, Yamagata, Japan). Two weeks after the inoculation, anti-mouse Gr-1 mAb were purified from ascites of SCID mice using ammonium sulphate treatment independently. Anti-mouse CD4 mAb were harvested from hybridoma cells (GK. 1. 5) and for anti-mouse CD8 mAb, we used hybridoma cells (53-6. 72) in the same methods. Rabbit anti-asialo GMI Ab was purchased from Wako Pure Chemicals (Osaka, Japan).

\section{Antibody treatment}

To deplete neutrophils, anti-Gr-1 mAb (400 $\mu$ g per mouse in each inoculation) was injected intraperitoneally 1 day before treatment and 1, 3, and 5 days after treatment. To examine the effect of anti-Gr-1 mAb administration on circulating neutrophils, the total number of peripheral white blood cells (WBC) was counted after staining with May-Grünwald Giemsa staining. For CD4+ or CD8+ $\mathrm{T}$ cell depletion, the mixture of each $100 \mu \mathrm{g}$ of anti-mouse CD4 mAb and antimouse CD8 mAb were inoculated intraperitoneally into mice 2 days before treatment and 0 and 3 and 6 days after treatment. To deplete NK cells, the mice were injected with anti-asialo GM 1 Ab intraperitoneally 1 day before treatment and 2 and 5 days after treatment. The depletion of these cells was confirmed in the splenocytes by flow cytometry, 2 days after treatment, and $95 \%$ of the appropriate subsets were constantly depleted.

\section{Induction of CNV}

PC induced CNV was generated by a previously described technique with some modifications. ${ }^{19}$ Briefly, the mice were anaesthetised with ketamine hydrochloride $(100 \mathrm{mg} / \mathrm{kg}$ body weight), and the pupils were dilated with $1 \%$ tropicamide. Krypton multicolour laser photocoagulation (wavelength $630 \mathrm{~nm}, 0.1$ second, spot size $100 \mu \mathrm{m}$, power $150 \mathrm{~mW}$ ) was performed around the disc of the retina through a slit lamp delivery system using a photocoagulator (MC7000; NIDEK, Aichi, Japan). The posterior pole of the retina was thus burned while a hand held cover slide was used as a contact lens. Only lesions in which a subretinal bubble or focal serous detachment of the retina developed were used for the experiments.

\section{Isolation and counting of the ocular infiltrating inflammatory cells}

To examine the ocular infiltrating cells in the retina and choroid, single cells were prepared from mouse eyes according to the procedure used to isolate of hepatic lymphocytes, with some modifications..$^{21}$ To collect a sufficient number of ocular infiltrating cells, 40-50 burns were delivered to the eyes of the mice to induce panretinal photocoagulation. After PC, the eyes were enucleated and then the anterior segment (cornea, iris, and lens) was taken out. The posterior segment of the eye including the sclera, choroid, retina was disrupted with scissors and then shaken in medium supplemented with $0.5 \mathrm{mg} / \mathrm{ml}$ of Collagenase type D (Boehringer Mannheim, Germany) at $37^{\circ} \mathrm{C}$ for 40 minutes. As the basic medium, we used RPMI 1640 (Gibco Laboratories, Grand Island, NY, USA) with $10 \%$ fetal bovine serum (Gibco), $100 \mathrm{U} / \mathrm{ml}$ penicillin and $100 \mu \mathrm{g} / \mathrm{ml}$ streptomycin, $\quad 5 \times 10^{-5} \mathrm{M} \quad$ 2-mercaptoethanol, $\quad 5 \mathrm{mg} / \mathrm{ml}$ HEPES buffer. The supernatants were collected, passed through a metal mesh, washed three times, and viable cells were thus obtained.

\section{Flow cytometry}

Intraocular infiltrating cells were adjusted to the designated concentrations and then were stained with the following regents and colours respectively. For identification of neutrophils, cells were stained with Cy-chrome conjugated anti-mouse CD45 mAb (30-F11) and FITC conjugated antimouse Ly-6G(Gr-1) mAb (RB6-8C5) and for macrophage identification, cells were stained with $\mathrm{Cy}$-Chrome conjugated anti-CD45 $\mathrm{mAb}$ and biotin conjugated anti-F4/80 mAb and then were counterstained by Streptavidin/R-PE were used. Cells were stained with Cy-chrome conjugated anti-mouse TCR $\beta m A b$ (H57-597) for T lymphocyte identification and were stained with FITC conjugated anti-mouse CD19 mAb (MB19-1) for B lymphocytes identification. NK cells were identification by staining with biotin conjugated anti-mouse NK1. I mAb (PK136) and then were counterstained by Streptavidin/R-PE. Flow cytometry was performed with EPICS XL (Beckman Coulter, Mannheim, Germany). The number of ocular infiltrating cells was calculated from the percentage of each population in the gate of the precounted total number of viable cells using trypan blue dye exclusion.

\section{Choroidal flat mount preparation}

In a mouse experimental CNV model, neovascularisation can be detected within 1 week. ${ }^{19}$ To study the effect of macrophage infiltration on the development of $\mathrm{CNV}$, we evaluated the early phase of $\mathrm{CNV}$ on day 7 using the flat mount technique as previously described. ${ }^{22}$ To individually evaluate the size of the CNV lesions, four burns were performed while leaving a space $(3,6,9,12$ o'clock positions around the optic disc). The mice were anaesthetised and perfused with $1 \mathrm{ml}$ of phosphate buffered saline containing $50 \mathrm{mg} / \mathrm{ml}$ of fluorescent labelled dextran (25 000 average molecular weight; Sigma, St Louis, MO, USA) and the eyes were removed and fixed for 30 minutes in $4 \%$ paraformaldehyde. The cornea and lens were removed and then the entire retina was carefully dissected from the eye cup. Radial cuts (average 8) were made from the edge of the eye cup to the equator and then the eyecup was flat mounted in an aqua-mount with the sclera facing down and the choroid facing up. Flat mounts were examined by fluorescence microscopy and the images were digitised using a three charge coupled device colour video camera and a frame grabber. To measure the total area of hyperfluorescence associated with each burn corresponding to the total number of fibrovascular scars, the MacScope (version 2. 3; Mitani, Fukui, Japan) was used.

\section{Statistics}

We used the statistical software StatView version 4.5 (Abacus Concepts Inc, Berkeley, CA, USA). A one way analysis of variance (ANOVA) was used to evaluate any differences in the numbers of ocular infiltrating inflammatory cells per eye. Multiple comparisons between each possible pair of groups were also evaluates by the Bonferroni's methods. A two sided p value less than 0.05 was considered statistically significant. The areas of CNVs were analysed to identify any significant differences among the experimental groups by Student's $t$ test. A value of $\mathrm{p} \leqslant 0.05$ was considered to be significant.

\section{RESULTS}

The kinetics of each subset of ocular infiltrating cells after photocoagulation

PC caused a disruption of the retinal pigment epithelium layer, Bruch's membrane, and choroidal vessels. Thereafter, many inflammatory cells were accumulated at the site of PC. $^{23}$ To analyse the phenotype of ocular infiltrating cells, single cells were prepared from the PC injured eyes by the treatment of collagenase as described in Materials and methods. The cells were analysed by flow cytometry and 


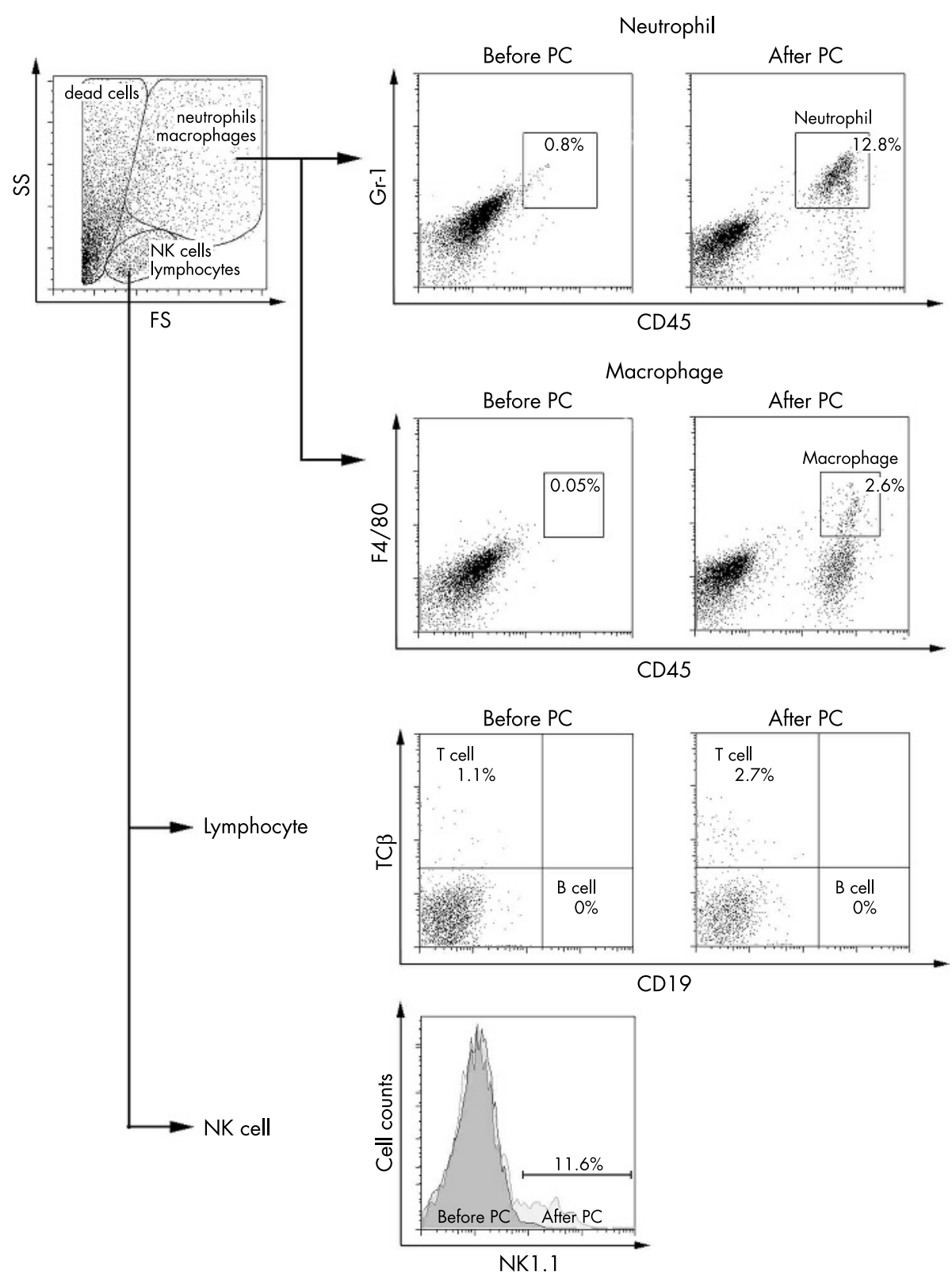

Figure 1 A representative FACS analysis of ocular infiltrating cells before $v$ after PC. A flow cytometric analysis was carried out on each ocular infiltration inflammatory cells. Left panel: The discrimination of ocular infiltrating cells by forward and side scattered gate. Only viable cells were analysed in the live gate. Typical neutrophil and macrophage gate and NK cell and lymphocyte gate were indicated. Right panel: Neutrophil and macrophage: The rectangular gates indicated neutrophils and macrophages. Neutrophils were stained with FITC conjugated anti-Gr-1 mAb and Cychrome conjugated anti-CD45 mAb. Macrophages were stained with biotin conjugated anti-F4/80 monoclonal antibody (mAb) counterstained by Streptavidin/R-PE and Cy-chrome conjugated anti-CD45 mAb. Lymphocyte: The quadrant gate indicated T cells and B cells. Cells were stained with Cy-chrome conjugated anti-mouse TCR $\beta$ chain $\mathrm{mAb}$ (T cells) and FITC conjugated anti-mouse CD19 mAb (B cells). NK cell: NK cells were indicated by the histogram. Cells were stained with and biotin conjugated anti-mouse NK1. 1 mAb counter stained by Streptavidin/R-PE.

only viable cells (excluding dead cells) were gated for further investigations (fig l, left panel). The discrimination of each subset of ocular infiltrating cells was primary performed based on forward and side scattered images (fig l, left panel) and then confirmed by the specific antibodies as described in materials and methods. Representative pattern of each subset (neutrophils, macrophages, lymphocytes and NK cells) were shown in figure 1, right panel. Neutrophil and macrophage gate and NK cell and lymphocyte gate were indicated. Specific gates were set up based on the staining pattern of isotype control antibodies.

In the neutrophils and macrophages gate, it contained large amounts of CD45 negative cells (fig 1, right panel). In the NK cells and lymphocytes gate, most of the cells were also CD45 negative cells (data not shown). We thought CD45 negative cells were not the bone marrow derived inflammatory cells and must be viable resident cells (including retinal pigment epithelial cells, glia cells, rod cells, cone cells and so on) dispersed by the collagenase treatment. In fact, these CD45 negative cells were observed even in the eye before PC (fig l, right panel).

After PC, as shown in figure 2, a large number of neutrophils and macrophages were infiltrated in the eye in early phase, peaked at 24 hours and 48 hours, respectively. A small amount of T cell and NK cell infiltration were detected. In this model, no B cells were detected throughout the entire 


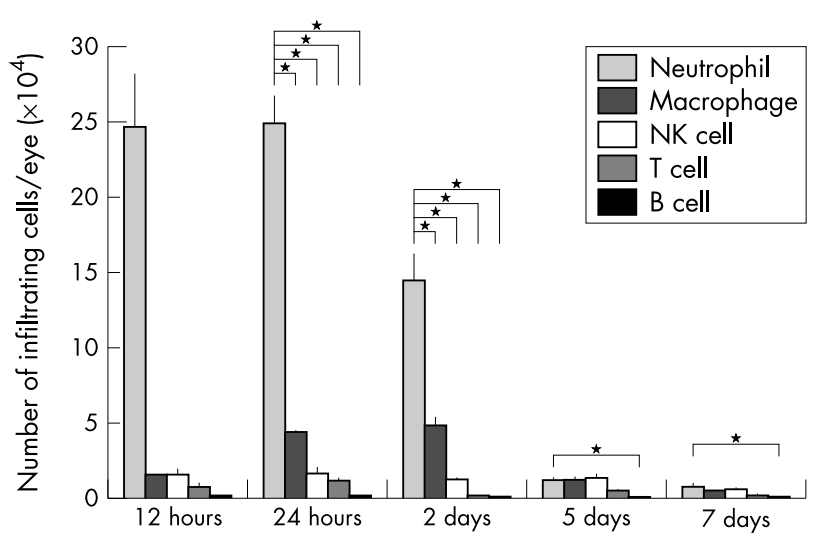

Figure 2 The kinetics of each subset of ocular infiltrating inflammatory cells after PC. The harvested cells from the murine eyes were analysed by flow cytometry at 12 hours, 24 hours, 2 days, 5 days, and 7 days after PC. The numbers of ocular infiltrating inflammatory cells per eye were calculated by the percentage of each population in the live gate and the precounted total number of viable cells using trypan blue dye exclusion. The gate of each antibody positive cell was set up according to the staining pattern of isotype control. These are the data of a representative series. We used 45 eyes in a series of kinetics experiments, and nine eyes were examined in individual time point $(n=3$, three eyes were pooled to obtain the designated cell numbers). We repeated this series three times and confirmed the similar results. The bars show the means (SEM) of three independent experiments for each time point $(n=3$ per time point for each individual experiment). ${ }^{*} \mathrm{p}<0.01$

disease course. Neutrophils were the major ocular infiltrating cells and, in fact, the amount of the infiltration of neutrophils was significantly larger than those of other cells at 12 hours, 24 hours, and 48 hours after PC. All of the bone marrow derived inflammatory cells were diminished until 7 days after PC.

\section{The effect of neutrophil depletion in CNV}

We have recently shown the role of macrophages in CNV formation. ${ }^{6}$ In this study, we found that not only macrophages but also a large number of neutrophils accumulated in the eye after PC. To examine the effect of neutrophils in the development of CNV, we thus depleted neutrophils in B6 mice by using anti-mouse Gr-1 Ab. Thereafter, the area of $\mathrm{CNV}$ was compared between the control B6 mice and antibody treated mice 7 days after PC. Although the area of $\mathrm{CNV}$ tended to decrease in Gr-1 mAb treated mice, no significant difference was observed compared to the nontreated control B6 mice (fig 3).

\section{The effect of either T lymphocyte or NK cell depletion in CNV formation}

In general, it is known that both T cells and NK cells have an important role in the initiation of inflammation. ${ }^{24}{ }^{25}$ We thus first examined the effects of $\mathrm{T}$ lymphocytes in $\mathrm{CNV}$ formation. T lymphocytes were depleted by the combination of anti-mouse CD4 mAb (GK 1.5) and anti-mouse CD8 mAb (53-6.72) in B6 mice. One day after mAbs treatment, PC was performed, and then the areas of CNVs were evaluated 7 days post-PC. Only slight differences were observed between the antibody treated group and the non-treated control group (fig 4A). This result suggested that the existence of $\mathrm{T}$ cells had no effect on CNV formation.

We next examined the role of NK cells in this model. We depleted NK cells using anti-asialoGMl Ab in B6 mice. There was no significant difference between anti-asialo GMl Abtreated B6 mice and non-treated control mice (fig 4B). We
A

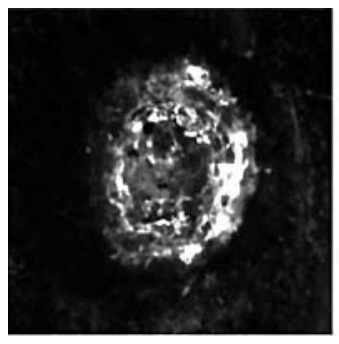

C57BL/6

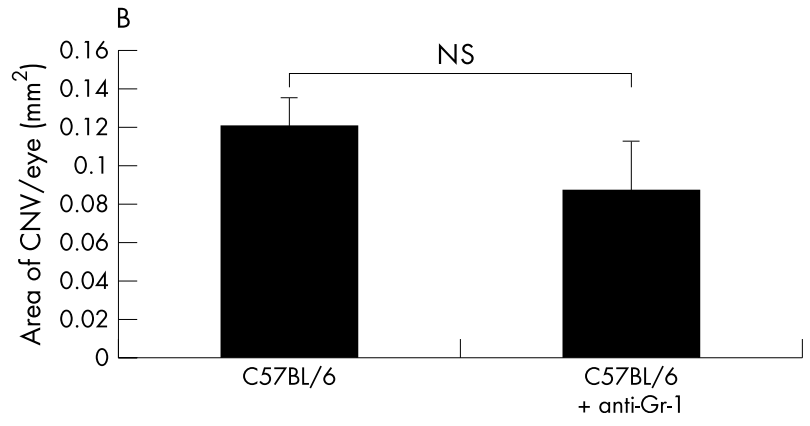

Figure 3 The effect of depletion of neutrophils in CNV formation. (A) The representative CNV lesions of choroidal flat mounts. Seven days after PC, the mice were perfused with fluorescein labelled dextran and the eyes were removed to make choroidal flat mounts. CNV was detected as a hyperfluorecsence vascular structure. The area of CNV was evaluated by a fluorescence microscope as described in Materials and methods. The photograph on the left shows typical CNV 7 days after $P C$ in C57BL/6 mice, and the photograph on the right shows CNV in C57BL/ 6 mice treated with an intraperitoneal inoculation of anti-Gr-1 $\mathrm{mAb}$. (B) The area of CNV per eye 7 days after PC in C57BL/6 mice treated with an intraperitoneal inoculation of anti-Gr-1 $\mathrm{mAb}(n=13)$ and control C57BL/ 6 mice $(n=22)$ were compared. The bars show the mean (SEM).

thus concluded that the NK cells had little effect on CNV development.

The effect of depletion of neutrophils in CCR2 KO mice CCR2 is the main receptor of C-C chemokine MCP-1, and it is strongly associated with macrophage migration to the particular lesions. ${ }^{26}{ }^{27}$ Therefore, macrophages could not migrate into any inflammatory lesions in CCR2 KO mice. ${ }^{28-30}$ We have previously observed a marked reduction in the number of ocular infiltrating macrophages and an impairment of CNV formation in CCR2 KO mice. ${ }^{6}$

Neutrophils have been reported to produce various angiogenic factors in several experimental models and they are thus considered to have a crucial role in angiogenesis associated disease. ${ }^{31}$ In addition, our result showed that the area of CNV in Gr-1 mAb treated mice had tendency to decrease compared to the non-treated control B6 mice (fig 3). We thus hypothesised that the contribution of macrophages was so strong that it and masked the angiogenic capacity of neutrophils in our model.

To confirm this hypothesis, we used CCR2KO mice and compared the CNV formation between anti-Gr-1 Ab treated mice and control CCR2KO mice. On day 7 after PC, a marked reduction in the $\mathrm{CNV}$ area was observed in CCR2KO mice compared to CCR2WT mice (C57BL/6 x 129Sv mice). Interestingly, the neutrophil depleted CCR2 KO mice significantly decreased the $\mathrm{CNV}$ area compared to the CCR2WT mice 7 days after PC (fig 5A,B). We thus concluded that although the macrophages are the main effector cells in CNV development, the neutrophils actually had an 
A
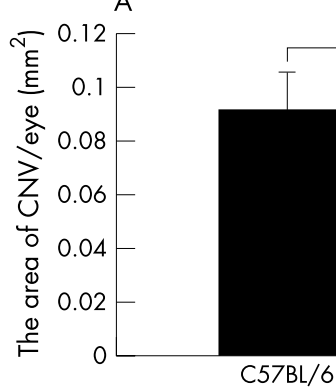

NS

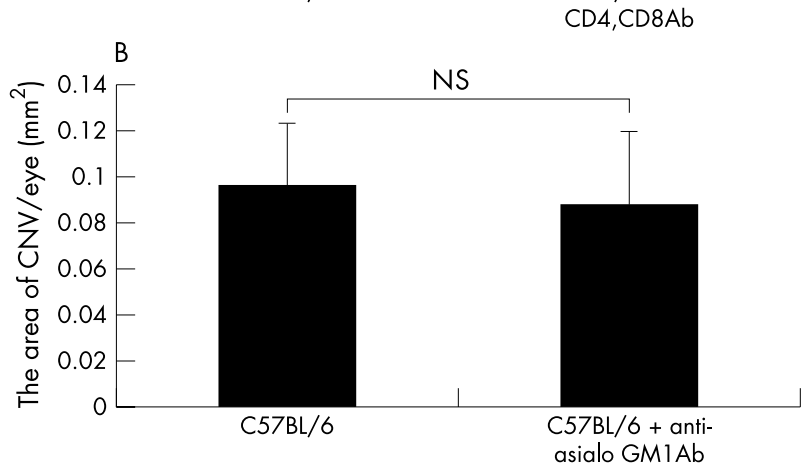

Figure 4 The effect of depletion of either T lymphocytes or NK cells in CNV formation. (A) The effect of T lymphocytes depletion in CNV formation. The area of CNV per eye in T lymphocytes depleted C57BL/6 mice treated with an intraperitoneal inoculation of anti-CD4 and CD8 $\mathrm{mAb}(\mathrm{n}=14)$ and control C57BL/6 mice $(n=12)$. The bars show the mean (SEM). (B) Comparison of the area of CNV in C57BL/6 mice treated with an intraperitoneal inoculation of anti-asialo GM-1 mAb $(n=8)$ and control C57BL/6 mice $(n=10)$.

angiogenic capacity and thereby partially contributed to CNV formation.

\section{DISCUSSION}

In this report, we examined the kinetics and relative contributions of various ocular infiltrated cells other than macrophages in the experimental CNV. We found that the NK cells and the lymphocytes were nothing to do with and the neutrophils partially contributed to CNV formation. Although it was shown that both NK cells and lymphocytes have a critical role in the initiation of the angiogenesis process in the tumour model, ${ }^{911}$ we think PC injured ocular tissues may strongly recruit neutrophils and macrophages, which participate in an innate immune responses, without the aid of T cells, B cells, and NK cells for CNV formation.

It is important to note that the angiogenic capacity of neutrophils actually existed but it was masked in the presence of macrophages, which are the dominant angiogenic promoter in this model. ${ }^{6}$ Our data indicated that neutrophil dependent choroidal angiogenesis could be dominant under special conditions that may impair the macrophage functions. Some investigators have reported that macrophages have a critical role on experimental CNV formation and they have suggested the therapeutic potential of macrophage depletion. ${ }^{32}{ }^{33}$ However, our data implied that macrophage depletion might induce the neutrophil dependent CNV.

In contrast with our data, several reports have shown that neutrophils and macrophages are equally contributed to angiogenesis in the other models. ${ }^{31}$ For instance, in a tumour $^{34}$ and skin wound healing model, ${ }^{35}$ neutrophils have been shown to be one of the main effector cells for angiogenesis. We assumed that the relative roles and dynamics of the local infiltrated cells could be altered

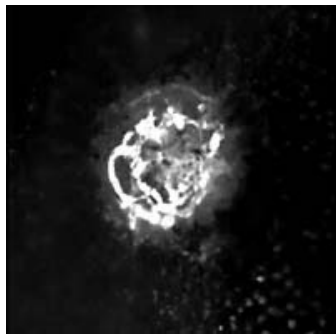

CCR2KO

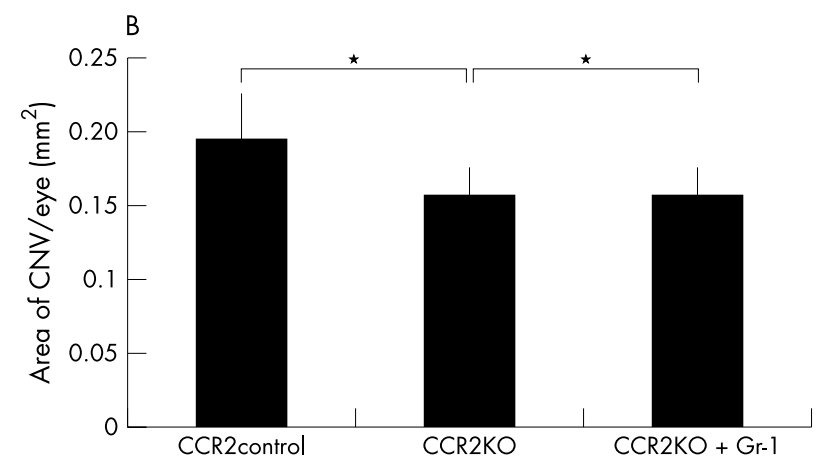

Figure 5 The effect of neutrophil depletion in CNV formation of CCR2 KO mice. (A) The representative CNV lesions of choroidal flat mounts. The area of CNV was evaluated as described in Materials and methods. The photograph on the left shows typical CNV 7 days after PC in CCR2KO mice, and the photograph on the right shows CNV in CCR2KO mice treated with an intraperitoneal inoculation of anti-Gr-1 mAb. (B) The area of CNV per eye were compared among CCR2WT mice (background: $\mathrm{C} 57 \mathrm{BL} / 6 \times 129 \mathrm{~Sv})(\mathrm{n}=18)$, no treated CCR2KO mice $(n=28)$ and $G r-1$ treated CCR2KO mice $(n=23) 7$ days after PC. The bars show the mean (SEM), ${ }^{*} p<0.01$.

depending on differences in the organs, tissue, and site. Especially, the immune inflammatory cell dynamics in the eye are unique, and also demonstrate tissue peculiarity because the eye is considered to be an immune privileged organ. ${ }^{36-38}$ Whether our results depend on tissue specificity or not, further study is still required.

We need to discuss the fact that partial CNV formation was observed even after the removal of neutrophils in CCR2 KO mice (fig 4). As a result, not only bone marrow derived ocular infiltrated cells but also resident cells such as retinal pigmented epithelium (RPE); fibroblasts and microglia are thus suggested to contribute to CNV. Some reports have shown that resident RPE cells directly produced various angiogenic factors and thus contribute to the pathogeneses of age related macular degeneration (AMD)..$^{39}$ Ocular infiltrated inflammatory cells may have a role in CNV by amplifying the angiogenic abilities of resident cells.

There was the discrepancy between the results of CNV size in figures 3 and 4 and figure 5 . In figures 3 and 4, CNV size in antibody no treated B6 mice ranged from $0.09-0.12 \mathrm{~mm}^{2}$ and in figure 5, CNV size in antibody no treated CCR2WTmice was $0.2 \mathrm{~mm}^{2}$. We considered this size discrepancy must the result of the strain difference. The background of CCR2KO mice is the hybrid of $\mathrm{C} 57 \mathrm{BL} / 6 \times 129 \mathrm{~Sv}$ mice, the CNV size of C57BL/6 $\times 129 S v$ mice (CCR2WTmice) is constantly lager than that of $\mathrm{C} 57 \mathrm{BL} / 6$ mice.

The process of CNV observed in the patients is not simple and must combine various steps and cellular events. ${ }^{41}{ }^{42}$ In addition, PC induced experimental CNV model has been thought to reflect merely the early phase of human CNV formation..$^{43}$ However, the inflammation is extremely important in the initial phase of $\mathrm{CNV}^{5}$ and we believe that our data 
may thus help to eventually prevent CNV formation in human disease in the future.

\section{ACKNOWLEDGEMENTS}

We thank Mr Brian Quinn for editorial assistance and Ms Michiyo Takahara for her valuable technical support. This work was in part supported by grants from the Ministry of Education, Science, Sports and Culture, Japan (B2, No 14770962, K-H Sonoda, B2, No 11470365. No 13470369, T Ishibashi) and the Japan National Society for the Prevention of Blindness (K-H Sonoda).

\section{Authors' affiliations}

C Tsutsumi-Miyahara, K-H Sonoda, H Qiao, T Oshima, T Murata

M Miyazaki, T Ishibashi, Department of Ophthalmology, Graduate School of Medical Sciences, Kyushu University, Fukuoka, Japan

K Egashira, M Ishibashi, Department of Cardiovascular Medicine, Graduate School of Medical Sciences, Kyushu University, Fukuoka, Japan

S Hamano, Department of Parasitology, Graduate School of Medical Sciences, Kyushu University, Fukuoka, Japan

I F Charo, San Francisco General Hospital, Gladstone Institution of Cardiovascular Division, University of California, San Francisco, CA 94143, USA

\section{REFERENCES}

1 Macular Photocoagulation Study Group. Argon laser photocoagulation for neovascular maculopathy. Five-year results from randomised clinical trials. Arch Ophthalmol 1991;109:1109-14.

2 Macular Photocoagulation Study Group. Five-year follow-up of fellow eyes of patients with age-related macular degeneration and unilateral extrafoveal choroidal neovascularization. Arch Ophthalmol 1993;111:1189-99.

3 Killingsworth MC, Sarks JP, Sarks SH. Macrophages related to Bruch's membrane in age-related macular degeneration. Eye 1990;4:613-21.

4 Dastgheib K, Green WR. Granulomatous reaction to Bruch's membrane in age-related macular degeneration. Arch Ophthalmol 1994;112:813-8.

5 Campochiaro PA. Retinal and choroidal neovascularization. J Cell Physio 2000; 184:301-10.

6 Tsutsumi C, Sonoda KH, Egashira K, et al. The critical role of ocular-infiltrating macrophages in the development of choroidal neovascularization. J Leukoc Biol 2003;74:25-32.

7 Jackson JR, Seed MP, Kircher $\mathrm{CH}$, et al. The codependence of angiogenesis and chronic inflammation. Faseb J 1997;1 1:457-65.

8 Jain RK, Koenig GC, Dellian M, et al. Leukocyte-endothelial adhesion and angiogenesis in tumors. Cancer Metastasis Rev 1996;15:195-204.

9 Melder RJ, Koenig GC, Witwer BP, et al. During angiogenesis, vascular endothelial growth factor and basic fibroblast growth factor regulate natural killer cell adhesion to tumor endothelium. Nat Med 1996;2:992-7.

10 Benelli R, Morini M, Carrozzino F, et al. Neutrophils as a key cellular target for angiostatin: implications for regulation of angiogenesis and inflammation. Faseb J 2002;16:267-9.

11 Chen WS, Kitson RP, Goldfarb RH. Modulation of human NK cell lines by vascular endothelial growth factor and receptor VEGFR-1 (FLT-1). In Vivo 2002; 16:439-45

12 Melter M, Reinders ME, Sho M et al. Ligation of CD40 induces the expression of vascular endothelial growth factor by endothelial cells and monocytes and promotes angiogenesis in vivo. Blood 2000;96:3801-8.

13 Lutty GA, Liu SH, Prendergast RA. Angiogenic lymphokines of activated T-cell origin. Invest Ophthalmol Vis Sci 1983;24:1595-601.

14 Numasaki M, Fukushi J, Ono $M$, et al. Interleukin-17 promotes angiogenesis and tumor growth. Blood 2003;101:2620-7

15 El-Sabban ME, Merhi RA, Haidar HA, et al. Human T-cell lymphotropic virus type 1-transformed cells induce angiogenesis and establish functional gap junctions with endothelial cells. Blood 2002;99:3383-9.
16 Boring L, Gosling J, Cleary M et al. Decreased lesion formation in CCR2-/mice reveals a role for chemokines in the initiation of atherosclerosis. Nature 1998;394:894-7.

17 Tepper RI, Coffman RL, Leder P. An eosinophil-dependent mechanism for the antitumor effect of interleukin-4. Science 1992;257:548-51.

18 Watanabe K, Noda K, Hamano S, et al. The crucial role of granulocytes in the early host defense against Strongyloides ratti infection in mice. Parasitol Res 2000;86:188-93

19 Tobe T, Ortega S, Luna JD, et al. Targeted disruption of the FGF2 gene does not prevent choroidal neovascularization in a murine model. Am J Pathol 1998;153:1641-6

20 Hiromatsu K, Matsuzaki G, Tauchi Y, et al. Sequential analysis of T cells in the liver during murine listerial infection. J Immunol 1992;149:568-73.

21 Sonoda KH, Sakamoto T, Yoshikawa $\mathrm{H}$, et al. Inhibition of corneal inflammation by the topical use of Ras farnesyltransferase inhibitors: selective inhibition of macrophage localization. Invest Ophthalmol Vis Sci 1998;39:2245-51.

22 Mori K, Duh E, Gehlbach P, et al. Pigment epithelium-derived factor inhibits retinal and choroidal neovascularization. J Cell Physiol 2001;188:253-63.

23 Ishibashi T, Hata Y, Yoshikawa $\mathrm{H}$, et al. Expression of vascular endothelia growth factor in experimental choroidal neovascularization. Graefes Arch Clin Exp Ophthalmol 1997;235:159-67.

24 Badgwell B, Parihar R, Magro C, et al. Natural killer cells contribute to the lethality of a murine model of Escherichia coli infection. Surgery 2002; 132:205-12

25 Korsgren M. NK cells and asthma. Curr Pharm Des 2002;8:1871-6.

26 Furie MB, Randolph GJ. Chemokines and tissue injury. Am J Pathol 1995:146:1287-301.

27 Strieter RM, Standiford TJ, Huffnagle GB, et al. "The good, the bad, and the ugly. "The role of chemokines in models of human disease. J Immunol 1996; 156:3583-6.

28 Boring L, Gosling J, Chensue SW, et al. Impaired monocyte migration and reduced type 1 (Th1) cytokine responses in C-C chemokine receptor 2 knockout mice. J Clin Invest 1997; 100:2552-61.

29 Kurihara T, Warr G, Loy J, Bravo R. Defects in macrophage recruitment and host defense in mice lacking the CCR2 chemokine receptor. J Exp Med 1997; 186:1757-62.

30 Kuziel WA, Morgan SJ, Dawson TC, et al. Severe reduction in leukocyte adhesion and monocyte extravasation in mice deficient in CC chemokine receptor 2. Proc Nat Acad Sci USA 1997;94:12053-8.

31 Shaw JP, Chuang N, Yee H, Shamamian P. Polymorphonuclear neutrophils promote rFGF-2-induced angiogenesis in vivo. J Surg Res 2003;109:37-42.

32 Espinosa-Heidmann DG, Suner IJ, Hernandez EP, et al. Macrophage depletion diminishes lesion size and severity in experimental choroidal neovascularization. Invest Ophthalmol Vis Sci 2003;44:3586-92.

33 Sakurai E, Anand A, Ambati BK, et al. Macrophage depletion inhibits experimental choroidal neovascularization. Invest Ophthalmol Vis Sci 2003:44:3578-85

34 Zhang H, Issekutz AC. Growth factor regulation of neutrophil-endothelial cell interactions. J Leukoc Biol 2001;70:225-32.

35 Gillitzer R, Goebeler M. Chemokines in cutaneous wound healing. J Leukoc Biol 2001;69:513-21.

36 Streilein JW. Regulation of ocular immune responses. Eye 1997;11:171-5.

37 Streilein JW, Stein-Streilein J. Does innate immune privilege exist? J Leukoc Biol 2000;67:479-87.

38 Streilein JW, Ohta K, Mo JS, et al. Ocular immune privilege and the impact of intraocular inflammation. DNA Cell Biol 2002;21:453-9.

39 Grossniklaus HE, Ling JX, Wallace TM, et al. Macrophage and retinal pigment epithelium expression of angiogenic cytokines in choroidal neovascularization. Mol Vis 2002;8:119-26.

40 Yu MJ, Shen WY, Lai MC, et al. The role of vascular endothelial growth factor (VEGF) in abnormal vascular changes in the adult rat eye. Growth Factors 2000;17:301-12.

41 Green WR, Enger C. Age-related macular degeneration histopathologic studies. The 1992 Lorenz E Zimmerman Lecture. Ophthalmology 1993; 100:1519-35.

42 Green WR. Histopathology of age-related macular degeneration. Mol Vis 1999;5:27

43 Ishibashi T, Miller H, Orr G, et al. Morphologic observations on experimental subretinal neovascularization in the monkey. Invest Ophthalmol Vis Sci 1987;28:1116-30. 